\title{
A ROAD EXTRACTION METHOD BASED ON HIGH RESOLUTION REMOTE SENSING IMAGE
}

\author{
Zhang Xia, Zhang Chunkang, Li Hongmei, Luo Zhu \\ The Mining College of Guizhou University, Guiyang, China, 2788722658@qq.com
}

KEY WORDS: high-resolution remote sensing images; mean shift; Stroke Width Transform(SWT); road extraction; median filter

\begin{abstract}
:
Aiming at the road extraction in high-resolution remote sensing images, the stroke width transformation algorithm is greatly affected by surrounding objects, and it is impossible to directly obtain high-precision road information. A new road extraction method combining stroke width transformation and mean drift is proposed. In order to reduce road holes and discontinuities, and preserve better edge information, the algorithm first performs denoising preprocessing by means of median filtering to the pre-processed image. Then, the mean shift algorithm is used for image segmentation. The adjacent parts of the image with similar texture and spectrum are treated as the same class, and then the fine areas less than the maximum stroke width are reduced. On the basis , the road information is extracted by the stroke width transformation algorithm, and the information also contains a small amount of interference information such as spots (non-road). In order to further improve road extraction accuracy and reduce speckle and non-road area interference, the basic operations and combinations in mathematical morphology are used to optimize it. The experimental results show that the proposed algorithm can accurately extract the roads on high-resolution remote sensing images, and the better the road features, the better the extraction effect. However, the applicability of the algorithm is greatly affected by the surrounding objects.
\end{abstract}

\section{Introduction}

With the improvement of the resolution of remote sensing images, the use of high-resolution images for road information extraction has important application value in car navigation, traffic management, urban planning, geographic information system database updating, and production of electronic maps. It is a hot topic for scholars at home and abroad. The appearance of high-resolution images, the more detailed presentation of the geometric spectral features of the road, which increases the possibility of accurately extracting road information from remote sensing images, but on the other hand, redundant details such as houses, barriers, shadows, vehicles All other factors will cause huge interference to road extraction, which in turn increases the difficulty of road extraction algorithm design ${ }^{[1]}$. The existing methods of road extraction from high-resolution images can be basically divided into three categories. One is based on road region extraction method, which recognizes and extracts road information according to some characteristics of the region in a certain target, including mathematical morphology ${ }^{[2]}$, Hough transform ${ }^{[3]}$, etc. The second is based on the method of extracting the edge of the road. The main principle of the method is to detect the gray point of the image and mark it as an edge according to certain criteria. Representative methods include edge detection operator $\operatorname{method~}^{[4,5]}$, Snake model method ${ }^{[6]}$, etc. The third is based on road center line extraction method, which needs to manually set the initial direction and target point to track the road and extraction, its representative method is the template matching $\operatorname{method}^{[7,8]}$. Due to the influence of various factors in the imaging process of remote sensing image, The phenomenon that the same object presents different spectra and different objects present the same spectra exists in the image, which increases the use of a single algorithm to extract multiple types 
of road limitations, and combines different algorithms to extract road information. Become more feasible. Therefore, this paper proposes a high-resolution remote sensing image road extraction combined with stroke width transformation and mean shift.

The mean shift algorithm is widely used in image segmentation because it does not require prior knowledge and does not have the advantage of pre-specifying the number of clusters. On the basis of the research on image segmentation algorithm, Zhang $\mathrm{L} \mathrm{Y}^{[9]}$ et al. proposed a road extraction algorithm combining mean shift and using statistical area to remove and merge small regions, and achieved good results. $\mathrm{Li} \mathrm{H} \mathrm{S}^{[10]}$ et al. aiming at the unsatisfactory effect of road information extraction from remote sensing images using threshold segmentation, a method combining Mean Shift with threshold segmentation is proposed to extract road information, which is better in images with simple objects and less interference. The Stroke Width Transform (SWT) was originally proposed for text detection, and is mostly used for text detection of natural scenes ${ }^{[11]}$. There are few studies on road extraction. Hou Y Y ${ }^{[12]}$ et al applied the stroke width transformation algorithm to the road extraction of UAV Image, and combined with the K-means clustering algorithm to achieve accurate road detection. Hailing Zhou ${ }^{[13]}$ et al. proposed a method for automatically detecting roads in aerial images of drones. In the seed selection part of the method, the stroke width transformation algorithm and the Gaussian mixture model are introduced. Based on this, a convex active contour model segmentation method is proposed to extract the entire road area. Therefore, based on the stroke width transformation algorithm, this paper proposes to improve the road extraction accuracy by combining the mean shift algorithm. Finally, the morphological processing is used to remove the interference and burr, and the road is accurately extracted.

\section{Road extraction method combined with stroke width} transformation and mean shift

\subsection{Stroke Width Transform(SWT)}

SWT was originally introduced as a pre-processing step for text detection because the stroke width of text characters has a certain stability, while non-text characters have a very large change in stroke width, so the difference in stroke width between the two is Very high ${ }^{[14]}$. SWT is a partial image operation that calculates the most likely stroke width value for each pixel based on the color information of each pixel in the image $^{[15]}$. The specific steps are as follows:

1) Establishing a width image having the same size as the original image, and setting the stroke width value of each pixel in the width image to infinity under initial conditions;

2) Performing edge detection on the image by using the canny operator to calculate the gradient direction of the edge point;

3) Starting from any edge point $P$, according to the gradient direction $d_{p}$ of the point, search for another edge point $q$ corresponding to it along the ray $r=p+n * d p(n>=0)$, if the gradient direction $d_{q}$ of the point $q$ is found to be exactly opposite to the search direction $d_{p}\left(d_{p}=d_{q} \pm \frac{\pi}{6}\right)$, then, the spatial position distance between the point $\mathrm{p}$ and the point $\mathrm{q}$, that is, the Euclidean distance $\|p-q\|$ is assigned to the value of all the pixels on the line connecting the two points in the width image.

4) Each pixel in the above process may be given the stroke width value more than once, so each new value should be compared with the original value, taking a smaller value, that is, the stroke width of the final record of each pixel. The value is always the width of the thinnest stroke it corresponds to.

\subsection{Mean Shift algorithm}

The mean shift algorithm is a simple and effective iterative clustering method, which is a nonparametric method based on probability density gradient estimation. The core idea is to estimate the clustering behavior of feature space sample points, and search for the target position of the sample point in the feature space by iterative operation, that is, the position with the highest probability density, then move the sample point to the target position and repeat the iterative process. Until all sample points are included in a certain part ${ }^{[16]}$.

ChenY ${ }^{[17]}$ extended the mean shift algorithm, which introduced the concept of kernel functions and bandwidth matrices. Let the sample set $X$ be obtained in n-dimensional space $d_{R}$ by n-time independent sampling of the probability density estimation function $\hat{f}(x)$, then:

$$
\hat{f}_{K}(x)=\frac{1}{n h^{d}} \sum_{i=1}^{n} K\left(\frac{x-x_{i}}{h}\right)
$$

Where $h$ is the bandwidth parameter, the kernel function 
$\left.K(X)=k\|x\|^{2}\right)$. Then the mean shift vector is $M_{H_{i}}(x)=m H_{i}(x)_{-x}$. Let the weights of the sampling points be equal, $g(x)=-k^{\prime}(x)$, and the iterative formula for the mean shift is:

$$
x_{i+1}=m H_{i}(x)=\frac{\sum_{i=1}^{n} g\left(\left\|\left(x-x_{i}\right) h^{-1}\right\|^{2}\right) x_{i}}{\sum_{i=1}^{n} g\left(\left\|\left(x-x_{i}\right) h^{-1}\right\|^{2}\right)}
$$

The probability density at the weighted average $m_{H_{i}}(x)$ of the sample points in the equation is generally greater than the probability density at $x$.

When the image is segmented by the mean shift algorithm, the sample points of the feature space are image data points, the image data is combined with the color information and the spatial position information in the description of the feature space, so it can be described by vectors $x=\left(x^{s}, x^{r}\right)$. Where $x^{s}$ is the position information of the sample point, which $x^{r}$ is the color information of the sample points. Then the multidimensional kernel function can be expressed as:

$$
K_{h_{S}, h_{r}}(x)=\frac{C}{h_{S}^{2} h p} k\left(\left\|\frac{x^{s}}{h_{S}}\right\|^{2}\right) k\left(\left\|\frac{x^{r}}{h_{r}}\right\|^{2}\right)
$$

Where

$$
\begin{aligned}
& h_{s}=\text { the spatial bandwidth coefficient } \\
& h_{r}=\text { the color bandwidth coefficient } \\
& K=\text { the kernel function } \\
& C=\text { the constant } \\
& p=\text { the dimension of the color information. }
\end{aligned}
$$

\subsection{Road extraction combined with stroke width transformation and mean shift}

High-resolution remote sensing images always have "almost" homogenous elongated regions of "more or less" constant width, and the road edges change slowly, which means that the variation of color and width of individual roads is limited. The stroke width transformation of the image calculates the stroke width value for each pixel, with the ability to clearly show the change in road width. The width distribution of the road area is different from the width distribution of other environments so that the SWT can indicate the road area. Therefore, this paper proposes the use of stroke width transformation algorithm to extract roads from high-resolution remote sensing images. However, it is found from the implementation of SWT algorithm that the extraction accuracy of SWT has a great dependence on the results of Canny edge detection. The Canny edge detector is sensitive to noise around the target object, and it is easy to detect many small non-road edges, and the interference is large. In addition, when the SWT is extracted from the road, the width of all the extracted information is smaller than the maximum stroke width value, in addition to the road information, there are non-road information smaller than the maximum stroke width value, such as green belts, trees, and buildings on both sides of the road, as well as some rivers, parking lots, hardened open spaces, etc., are highly likely to be mistaken for roads due to their similar characteristics to roads and widths less than the maximum stroke width value Therefore, using the SWT algorithm alone will inevitably extract non-road information and affect the road extraction accuracy.

In order to solve the above problems, effectively extract the road information in the image and improve the final extraction precision of SWT, a road extraction algorithm based on stroke width transform and mean shift is proposed. Firstly, the pre-processed image is denoised by means of median filtering. Since both mean shift and SWT need to use boundary information, median filtering is chosen to denoise, which has better edge protection. The spectral and texture features of roads and other features in the median filtered image become more balanced, which can reduce the hole and discontinuity of the road in the image after the mean shift segmentation, so that it has a more complete segmentation result. When performing mean shift partitioning, the partitioning scale is controlled by controlling spatial bandwidth parameters and color bandwidth parameters. The bandwidth value determines the range of each iteration, therefore, the difference in bandwidth causes the high-dimensional sphere area in the process to contain different numbers, distributions, and densities of pixels. By selecting the appropriate bandwidth parameters, the Mean Shift algorithm can achieve better image segmentation accuracy. After the image is segmented by the mean shift algorithm, the adjacent parts of the image with similar texture and spectrum are divided into the same class, which reduces the fine-grained area smaller than the maximum stroke width value. On this basis, the segmented image is used as the input image of SWT, and the optimal maximum stroke width value is determined experimentally to extract the road. The mean shift is added to 
improve the SWT algorithm to reduce the extraction of non-road areas, so that the information extracted by the stroke width transformation contains fewer non-road areas. At this time, the extracted road information still contains a small amount of interference information, in order to further improve the accuracy of road extraction, the basic operations and combinations in mathematical morphology are used to optimize the processing, among them, the combination of corrosion and expansion is used to eliminate the spots and filling voids in the extraction result. and according to the size of the noise area in the image, selects an appropriate threshold to remove the noise, and then removes the burr and refines the process to obtain the final road image, and extracts the result, and the accuracy of the extraction result is evaluated. The algorithm flow is shown in figure 1:

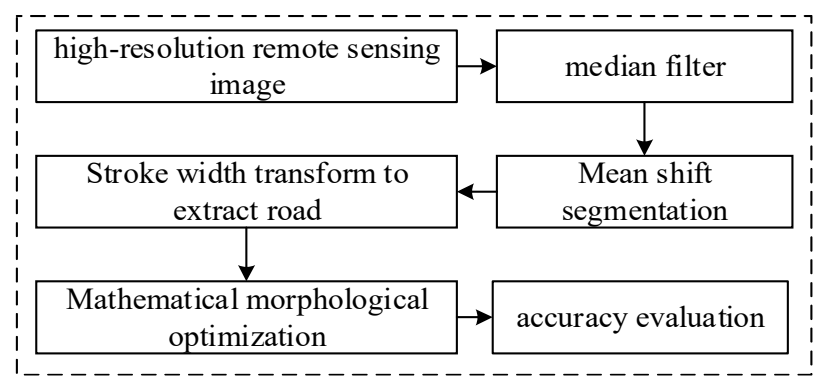

Figure 1. Road information extraction process

\section{Experiment and analysis}

In this paper, using Matlab2016a, the high-resolution 2 optical satellite images is used as the experimental data, and the road extraction algorithm based on stroke width transformation and mean shift is realized. The feasibility of the proposed algorithm is verified by the following experiments, and compared with the direct use of SWT algorithm to verify the accuracy of the proposed algorithm.

The accuracy of road extraction is quantitatively evaluated by three indicators, namely completeness, accuracy, and quality ${ }^{[18]}$, expressed by $C P, C R$, and $Q L$, respectively.

$$
C P=\frac{T N}{F N} ; \quad C R=\frac{T P}{F P} ; \quad Q L=\frac{T P}{F P+L P}
$$

Where $\quad T N=$ the matching road length

$F N=$ the actual road length

$T P=$ the matching road length obtained during the extraction process

$F P=$ the total length of the extraction process

$L P=$ the unmatched road length
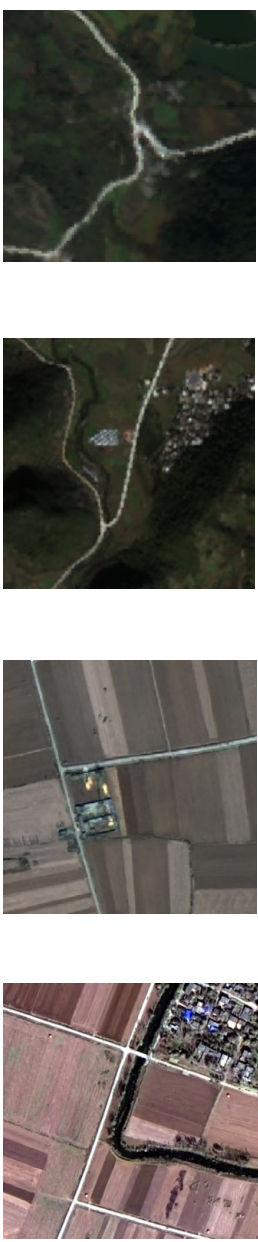

experiment 4

(The first column is the original image, the second column is the extract result graph of the new algorithm, the third column is the extract result graph of SWT.)

The white area in the figure is the extracted road information. By comparing the above four groups of experiments, the results of the algorithm are accurate, and the road information is relatively complete, but there are also some broken parts, which are caused by the occlusion of the surrounding objects; the road extracted directly by SWT algorithm Information, because there are non-road slender objects similar to the width of the road in the image, there is a large area of wrong extraction information in the final result;

The algorithm extraction effect of this paper is better than the direct SWT extraction. This is because the SWT algorithm is sensitive to background noise. It is easy to detect a lot of redundant information for the background complex image, and there is a big error extraction problem. Combine mean shift and SWT. It is a good way to make up for the above problems, and the extracted road edges are better, the burrs are less, and the road information is extracted accurately. It is proved that the 
proposed algorithm can not only extract the road, but also effectively suppress the noise, reduce the error extraction and leakage extraction, and the accuracy is higher than the classic SWT algorithm.

\begin{tabular}{llll}
\hline \multicolumn{1}{c}{ Experiment } & $\mathrm{CP}(\%)$ & $\mathrm{CR}(\%)$ & $\mathrm{QL}(\%)$ \\
\hline Experiment 1 new algorithm & 91.389 & 91.695 & 86.648 \\
Experiment 1 SWT & 87.379 & 61.584 & 57.796 \\
Experiment 2 new algorithm & 94.863 & 96.256 & 94.684 \\
Experiment 2 SWT & 92.971 & 92.914 & 86.812 \\
Experiment 3 new algorithm & 95.295 & 96.072 & 94.398 \\
Experiment 3 SWT & 81.009 & 85.720 & 71.376 \\
Experiment 4 new algorithm & 92.717 & 84.147 & 78.777 \\
Experiment 4 SWT & 82.038 & 63.521 & 55.765 \\
\hline
\end{tabular}

Table 1. algorithm accuracy

Analysis Table 1, the integrity of the road extraction results is higher than the integrity of the road extraction results directly using SWT, indicating that the method can better identify the road area; the road extraction results are more correct, indicating The method can extract the road area more correctly; the quality index of the road extraction result is higher, indicating that the method can effectively improve the road extraction quality. It can be seen that the proposed algorithm has better extraction accuracy for high resolution remote sensing image roads.

\section{Conclusion}

Based on the research of road extraction algorithm, this paper proposes a new algorithm for extracting roads from high resolution remote sensing images combined with stroke width transformation and mean shift. Aiming at the problem that SWT extraction accuracy is not high, the advantages of median filtering and mean shift segmentation are used to improve the accuracy of SWT in road extraction. The validity and accuracy of the algorithm are verified by comparison with classical SWT algorithm and objective evaluation. The proposed algorithm can accurately extract the roads on high-resolution remote sensing images, and the road extraction accuracy is significantly improved. Inadequacies: The algorithm of this paper is effective for linear features and simple background extraction. However, the algorithm is not applicable to roads with complicated backgrounds and high and complex surrounding structures. The maximum stroke width value in the stroke width transformation has a direct impact on the road extraction quality, if it can be automatically based on the width information of the roads in the image. Determining the best value of this value will further improve the automation of the algorithm, which will be the focus of the next step.

\section{References}

[1] ZHU E Z, SONG W D, DAI J G. Road extraction of high-resolution remote sensing images based on Improved SVM[J]. Science of Surveying and Mapping, 2016, 41(12): 224-228.

[2] ZHOU Q L. The Research of road extraction from high-resolution remote sensing takes into account of geometric features and mathematical morphology[D]. Central South University, 2013.

[3] LIU X D, LIU Y. Urban road extraction from remote sensing image based on Hough transform and mathematical morphology[J]. Journal of Nanjing Normal University(Natural Science Edition), 2010, 33(04): 128-133.

[4] HUANG W, HUANG H X, XU J M, et al. An improved road extraction method for remote sensing images based on Canny edge detection[J]. Remote Sensing for Land \& Resources, 2019, 31(01): 65-70.

[5] TAN Y, HUANG H X, XU J M, et al. Road edge detection from remote sensing image based on improved Sobel operator[J]. Remote Sensing for Land \& Resources, 2016, 28(03): 7-11.

[6] FU X Y, ZHANG F L, WANG G J, et al. Road extraction from SAR images using tensor voting and Snakes model[J]. Journal of Image and Graphics, 2015, 20(10): 1403-1411.

[7] LIN G X, ZHANG J X, LI H T,et al. Semi-automatic extraction of ribbon road from high resolution remotely sensed imagery by a T-shaped template matching $[\mathrm{J}]$. Geomatics and Information Science of Wuhan University, 2009, 34(03): 293-296.

[8] LIAN R B, WANG W X, LI J. Road extraction from high-resolution remote sensing images based on adaptive circular template and saliency map[J]. Acta Geodaetica et Cartographica Sinica, 2018, 47(07): 950-958.

[9] ZHANG Y L, SHAO Y D, YANG Y, et al. Road extraction from high resolution remote sensing images based on improved Mean Shift algorithm method[J]. Remote Sensing Information, 2010, (04): 3-7.

[10] LI S H, HUANG P P, SU Y. A method for road extraction from remote sensing imagery[J]. Remote Sensing for Land \& 
Resources, 2015, 27(02): 56-62.

[11] Andrej Ikica, Peer Peter. Influence of image quality on SWT voting-based color reduction method for detecting text in natural scene images[A]//EEE, 2014: 142-148.

[12] HOU Y Y, WANG H. Visual road detection from UVA images based on Stroke Width Transformation[J]. Computer \& Digital Engineering, 2018, 46(01): 182-186.

[13] H Zhou, Kong H, Wei L, et al. On Detecting Road Regions in a Single UAV Image[J]. IEEE Transactions on Intelligent Transportation Systems, 2017, 18; 18(7; 7): 1713-1722.

[14] EPSHTEIN B, OFEK E, WEXLER Y. Detecting text in natural scenes with stroke width transform[C]//IEEE Computer Society Conference on Computer Vision and Pattern Recognition, 2010: 2963-2970.

[15] CHEN S, ZHENG J B, ZHAN E Q, et al. Text detection based on stroke angle conversion and stroke width features in natural scene[J]. Application Research of Computers, 2019, (04): 1-2.

[16] WANG L G. Research on the high spatial resolution remote sensing image segmentation based on nonblurring mean shift [D]. Wuhan University, 2009.

[17] CHENG Y. Mean shift, mode seeking, and clustering[J]. IEEE Transactions on Pattern Analysis and Machine Intelligence, 1995,17; 17(8; 8):790-799.

[18] LI J. Study on information extraction method of main features in Bazhou Xinjiang area based on GF-2[D]. Beijing Forestry University, 2016.

Tel: 15685510443 